\title{
Dietary calcium decreases but short-chain fructo-oligosaccharides increase colonic permeability in rats
}

\author{
Marloes A. A. Schepens ${ }^{1,2}$, Anneke Rijnierse ${ }^{1,3}$, Arjan J. Schonewille ${ }^{1,2}$, Carolien Vink ${ }^{1,2}$, \\ Robert-Jan M. Brummer ${ }^{1,4}$, Linette E. M. Willemsen ${ }^{5}$, Roelof van der Meer ${ }^{1,2,3}$ \\ and Ingeborg M. J. Bovee-Oudenhoven ${ }^{1,2} *$ \\ ${ }^{1}$ TI Food and Nutrition, PO Box 557, 6700 AN Wageningen, The Netherlands \\ ${ }^{2}$ NIZO Food Research, PO Box 20, 6710 BA Ede, The Netherlands \\ ${ }^{3}$ Department of Human Nutrition, Wageningen University and Research Centre, Bomenweg 2, 6703 HD Wageningen, \\ The Netherlands \\ ${ }^{4}$ School of Health and Medical Sciences, Örebro University, Örebro, Sweden \\ ${ }^{5}$ Pharmacology and Pathophysiology, Utrecht Institute for Pharmaceutical Sciences, Utrecht University, PO Box 80 082, \\ 3508 TB Utrecht, The Netherlands
}

(Received 23 February 2010 - Revised 28 June 2010 - Accepted 30 June 2010 - First published online 9 August 2010)

\begin{abstract}
An increased intestinal permeability is associated with several diseases. Nutrition can influence gut permeability. Previously, we showed that dietary $\mathrm{Ca}$ decreases whereas dietary short-chain fructo-oligosaccharides (scFOS) increase intestinal permeability in rats. However, it is unknown how and where in the gastrointestinal tract $\mathrm{Ca}$ and scFOS exert their effects. Rats were fed a Western low-Ca control diet, or a similar diet supplemented with either Ca or scFOS. Lactulose plus mannitol and Cr-EDTA were added to the diets to quantify small and total gastrointestinal permeability, respectively. Additionally, colonic tissue was mounted in Ussing chambers and exposed to faecal water of these rats. Dietary Ca immediately decreased urinary Cr-EDTA excretion by $24 \%$ in Ca-fed rats compared with control rats. Dietary scFOS increased total Cr-EDTA permeability gradually with time, likely reflecting relatively slow gut microbiota adaptations, which finally resulted in a $30 \%$ increase. The lactulose:mannitol ratio was $15 \%$ higher for Ca-fed rats and $16 \%$ lower for scFOS-fed rats compared with control rats. However, no dietary effect was present on individual urinary lactulose and mannitol excretion. The faecal waters did not influence colonic permeability in Ussing chambers. In conclusion, despite effects on the lactulose:mannitol ratio, individual lactulose values did not alter, indicating that diet did not influence small-intestinal permeability. Therefore, both nutrients affect permeability only in the colon: Ca decreases, while scFOS increase colonic permeability. As faecal water did not influence permeability in Ussing chambers, probably modulation of mucins and/or microbiota is important for the in vivo effects of dietary $\mathrm{Ca}$ and scFOS.
\end{abstract}

Calcium: Short-chain fructo-oligosaccharides: Intestinal permeability: Diet

The intestinal epithelial cell layer constitutes the largest and most important barrier against the external environment. Tight contact between the enterocytes prevents access of intraluminal toxins, antigens and enteric microbiota to underlying tissue compartments. A compromised intestinal barrier function is suggested to be associated with the pathogenesis of a number of intestinal diseases including inflammatory bowel disease, coeliac disease, post-infectious irritable bowel syndrome and food allergy ${ }^{(1-4)}$. Accumulating evidence about the progression of inflammatory bowel disease, a chronic relapsing and remitting disease for which the aetiology remains unknown, shows that an increased intestinal permeability intensifies the exposure of the lamina propria to luminal contents, activating an abnormal immune response ${ }^{(3,5)}$.
Interestingly, nutrition can affect the epithelial barrier, and hence possibly influence disease development. We have shown in several controlled studies that dietary $\mathrm{Ca}$ protects against intestinal infections with foodborne pathogens ${ }^{(6-8)}$. $\mathrm{Ca}$ also has cytoprotective effects by precipitating cytotoxic surfactants, such as secondary bile acids ${ }^{(9,10)}$. In addition, supplemental $\mathrm{Ca}$ attenuates the development of colitis ${ }^{(11)}$. In the latter study, total intestinal permeability was quantified by the analysis of urinary Cr-EDTA excretion. We observed that supplemental $\mathrm{Ca}$ prevented the colitis-related increase in intestinal permeability in HLA-B27 transgenic rats ${ }^{(11)}$. Another example of dietary modulation of intestinal permeability is the supplementation of short-chain fructooligosaccharides (scFOS), which may be regarded as a prebiotic. Work from our group has shown that these dietary

Abbreviations: FITC-dextran, fluorescein isothiocyanate-labelled 4 kDa dextran; scFOS, short-chain fructo-oligosaccharides.

* Corresponding author: I. M. J. Bovee-Oudenhoven, fax +31 318650 400, email ingeborg.bovee@nizo.nl 
scFOS impair the resistance to intestinal infection in rats ${ }^{(8,12,13)}$. Subsequently, we showed that this decrease in barrier functioning was associated with a higher total intestinal permeability in rats ${ }^{(14)}$.

The dietary effects on intestinal permeability in the studies mentioned earlier were investigated by the addition of Cr-EDTA to the diet and subsequent measurement of urinary Cr-EDTA excretion. As Cr-EDTA is stable throughout the intestinal tract, the urinary excretion of this marker provides information regarding total intestinal permeability ${ }^{(15)}$. By choosing permeability probes that have limited exposure to certain parts of the intestinal epithelium, region-specific permeability measurements can be performed ${ }^{(16)}$. For example, the saccharide probes lactulose and mannitol can be used to measure small-intestinal permeability, because they are instantly degraded by the colonic microbiota. Since lactulose only passes the gut wall paracellularly and mannitol passes transcellularly, the lactulose:mannitol ratio is used to express small-intestinal permeability ${ }^{(2)}$.

We hypothesised that both a high-Ca diet and a scFOS diet mainly influence colonic permeability. As colitis is suggested to result from increased translocation of micro-organisms due to a higher colonic permeability and $\mathrm{Ca}$ attenuated colitis development $^{(11)}$, dietary $\mathrm{Ca}$ probably has its main effect on permeability of the colon. Since scFOS are fermented in the colon, where they change the luminal content, which possibly influences the colonic mucosa, we also hypothesised that scFOS mainly affect colonic permeability. The aim of the present study was to localise these effects. Therefore, we added Cr-EDTA, lactulose and mannitol as permeability markers to the rat diets. As no specific colonic permeability marker exists, this can be determined by comparing total (Cr-EDTA) and small (lactulose:mannitol ratio) intestinal permeability. To gain even more insight into the effect of dietary $\mathrm{Ca}$ and scFOS on permeability, we performed Ussing chamber experiments with the faecal waters derived from the in vivo study.

\section{Material and methods}

Experimental design of the nutritional study: animals and diets

The experimental protocol was approved by the Animal Welfare Committee of Wageningen University and Research Centre (Wageningen, The Netherlands). Specific pathogenfree outbred male Wistar rats (WU, Harlan, Horst, The Netherlands), 8 weeks old and with a mean body weight of $267 \mathrm{~g}$ at the start of the experiment, were housed individually in metabolic cages. Rats were maintained in temperature- and humidity-controlled environment in a $12 \mathrm{~h}$ light-dark cycle. Rats were fed ad libitum a purified 'humanised' Western diet which contained in the control situation (per kg): $200 \mathrm{~g}$ acid casein, $326 \mathrm{~g}$ maize starch, $162 \mathrm{~g}$ glucose, $160 \mathrm{~g}$ palm oil, $40 \mathrm{~g}$ maize oil, $50 \mathrm{~g}$ cellulose and $5 \cdot 16 \mathrm{~g} \mathrm{CaHPO} \mathrm{C}_{4} \cdot 2 \mathrm{H}_{2} \mathrm{O}$ (corresponding to $30 \mathrm{mmol} \mathrm{Ca} / \mathrm{kg}$ diet; Sigma-Aldrich, St Louis, MO, USA). In addition, the diets contained the following permeability markers per $\mathrm{kg}$ : $2 \mathrm{~g} \mathrm{Cr}$-EDTA, $7.5 \mathrm{~g}$ lactulose (Solvay Arzneimittel $\mathrm{GmbH}$, Hannover, Germany) and $2.5 \mathrm{~g}$ mannitol (Sigma-Aldrich) (see below). Lactulose and mannitol were added to the diet to avoid stressful supplementation by oral gavage which might have an impact on intestinal permeability and transit time. Moreover, the continuous exposure of the rats to the permeability probes excludes a possible day-night rhythm in intestinal permeability. Vitamins and minerals (other than $\mathrm{Ca}$ ) were added to both the diets according to AIN-93 ${ }^{(17)}$. This control diet was low in $\mathrm{Ca}$ and had a high fat content to mimic the composition of a Western human diet. The diet high in $\mathrm{Ca}$ contained $120 \mathrm{mmol} \mathrm{Ca} / \mathrm{kg}$ diet, and in the scFOS group, the control diet was supplemented with $60 \mathrm{~g} \mathrm{scFOS} / \mathrm{kg}$ diet (6\% (w/w); Raftilose ${ }^{\circledR} \mathrm{P} 95$, Orafti, Tienen, Belgium), both at the expense of glucose. The degree of polymerisation of the supplemented scFOS ranges between 2 and 8, and therefore they are classified as scFOS. Inert Cr-EDTA was added to the diets to quantify total intestinal permeability ${ }^{(18)}$. Cr-EDTA solution was prepared as described elsewhere and subsequently freeze dried ${ }^{(19)}$. To check the complete formation of the Cr-EDTA complex, the prepared Cr-EDTA solution was passed through a cation-exchange resin column (Chelex 100 Resin; Bio-Rad, Hercules, CA, USA). No uncomplexed $\mathrm{Cr}^{3+}$ ions were present. Potential binding and subsequent precipitation of Cr-EDTA to $\mathrm{Ca}$ were determined by quantification of the Cr-EDTA concentration in the faecal water. This was similar in the different dietary groups, so binding of Cr-EDTA to Ca was concluded to be absent. Small-intestinal permeability was assessed using lactulose and mannitol ${ }^{(2,20)}$. Food intake was recorded daily, and animal weight was recorded three times every week. After $14 \mathrm{~d}$ of experimental feeding, rats were anaesthetised with isoflurane and killed.

\section{Measurement of intestinal permeability}

Total $24 \mathrm{~h}$ urine samples were collected every $2 \mathrm{~d}$ during the experiment. For Cr-EDTA measurement, urine was acidified with $50 \mathrm{~g} / 1 \mathrm{TCA}$, centrifuged for $2 \mathrm{~min}$ at $14000 \mathrm{~g}$ and diluted with $0.5 \mathrm{~g} / \mathrm{l} \mathrm{CsCl}$. Then, $\mathrm{Cr}$ was analysed by inductively coupled plasma-atomic emission spectrophotometry. Urinary lactulose and mannitol were analysed by high-performance anion-exchange chromatography with pulsed amperometric detection on a $\mathrm{Au}$ electrode. The analyses were performed with a 600E System controller pump (Waters, Milford, MA, USA) with a He degassing unit and a model 400 EC detector (EG\&G, Princeton, NJ, USA). With a WPS-3000 autosampler (Dionex, Sunnyvale, CA, USA), $20 \mu$ l of the (diluted) sample were injected on a Carbopac MA-1, $250 \times 4 \mathrm{~mm}$, column (Dionex) thermostated at $30^{\circ} \mathrm{C}$. Lactulose and mannitol were eluted isocratically at a flow rate of $0.40 \mathrm{ml} / \mathrm{min}$ with $480 \mathrm{~mm}-\mathrm{NaOH}$ followed by a washing step of $1000 \mathrm{~mm}-$ $\mathrm{NaOH}$ and $1000 \mathrm{~mm}$-sodium acetate. Data analysis was done with Chromeleon software version 6.60 (Dionex).

\section{Ussing chambers}

All faeces were collected on the last $3 \mathrm{~d}$ of the nutritional experiment and freeze dried. Faecal water was prepared as described earlier ${ }^{(21)}$. The animal welfare committee of Utrecht University (Utrecht, The Netherlands) approved the animal protocol. Specific pathogen-free outbred male Wistar rats ( $n$ 6; WU), 7-8 weeks old, were killed by $\mathrm{CO}_{2}$ inhalation. The colon was taken out and washed in Ringer solution 
(25 mM-NaHCO 3 , 117.5 mM-NaCl, 1.25 mM-CaCl $2,5.7 \mathrm{~mm}-\mathrm{KCl}$, $1.2 \mathrm{mM}-\mathrm{NaH}_{2} \mathrm{PO}_{4}, 1.2 \mathrm{mM}-\mathrm{MgSO}_{4}$ and $5 \mathrm{~g}$ glucose, $\mathrm{pH} 7.4$ when gassed with $95 \% \mathrm{O}_{2}-5 \% \mathrm{CO}_{2}$ at $37^{\circ} \mathrm{C}$ ), and its muscle layers were stripped off. The tissue sections were cut into specimens of appropriate size and randomly mounted in Ussing chambers (manufactured by Technical Services of Utrecht Institute for Pharmaceutical Sciences), each side containing the Ringer solution. After a $30 \mathrm{~min}$ equilibration period, baseline transepithelial resistance was measured, and the Ringer solution in the mucosal compartment was replaced by fresh Ringer solution (negative control), or Ringer solution containing no $\mathrm{Ca}$, or faecal water prepared from faeces of the different dietary groups of the nutritional study described earlier. Colonic tissue was thus exposed to faecal water pools of either control rats, $\mathrm{Ca}$-fed rats or scFOS-fed rats. The $\mathrm{Ca}$ chelator ethylene glycol tetraacetic acid served as a positive control for enhanced permeability due to $\mathrm{Ca}$ binding $(8 \mathrm{~mm}$ as final concentration in Ringer solution in both compartments). At the same time, fluorescein isothiocyanate-labelled $4 \mathrm{kDa}$ dextran (FITC-dextran) was added at the mucosal side ( $1 \mathrm{mg} / \mathrm{ml}$ as final concentration). Samples were taken every $30 \mathrm{~min}$ from the serosal compartment for subsequent fluorescence measurement and replaced by fresh Ringer solution. The experiment was carried out for $180 \mathrm{~min}$, after which endline transepithelial resistance was determined. Flux was expressed as nanomol of FITC-dextran crossing $1 \mathrm{~cm}^{2}$ of colonic epithelium in $1 \mathrm{~h}\left(\mathrm{nmol} / \mathrm{cm}^{2}\right.$ per $\left.\mathrm{h}\right)$.

\section{Statistical analysis}

All the results are expressed as means with their standard errors. The predefined comparisons of interest for the nutritional study were control rats $v$. Ca-fed rats and control rats $v$. scFOS-fed rats. For the in vitro experiments, the predefined comparisons of interest were: faecal water of control rats $v$. faecal water of $\mathrm{Ca}$-fed rats, faecal water of control rats $v$. faecal water of scFOS-fed rats and the negative control $v$. withdrawal of $\mathrm{Ca}$ from the Ringer solution. Statistics were done by one-way ANOVA or Kruskal-Wallis test, depending on the normality of the data. If significant, this was followed by Student's $t$ test (for normally distributed data) or MannWhitney $U$ test (for non-normally distributed data) to identify the significant dietary effects. Differences were considered statistically significant when $P<0 \cdot 05$ (all two-sided). Statistical analyses were conducted with GraphPad Prism version 5.01 (GraphPad Software, Inc., La Jolla, CA, USA).

\section{Results}

\section{Animal growth and food intake}

Rats fed the high-Ca diet did not differ from rats fed the low-Ca control diet with respect to growth (5.8 (SE 0.3) and $6 \cdot 3(\mathrm{SE} 0 \cdot 3) \mathrm{g} / \mathrm{d}$, respectively; $P=0 \cdot 16)$. However, animal growth in the scFOS group was significantly lower (4.7 (SE $0 \cdot 3) \mathrm{g} / \mathrm{d}, P=0.0002)$ in accordance with a previous study ${ }^{(12)}$. Food intake of rats fed the low-Ca control diet and of rats fed the high-Ca diet was similar (21.6 (SE 0.4) and 21.4 (SE 0.5$) \mathrm{g} / \mathrm{d}$, respectively; $P=0.72$ ), while food intake of the scFOS-fed rats was significantly less than that of control rats (18.4 (SE 0.4) g/d, $P<0.0001)$.
Dietary calcium and short-chain fructo-oligosaccharides influence total intestinal permeability

Dietary Ca supplementation caused a direct and persistent decrease in urinary Cr-EDTA excretion; the decrease was $24 \%$ in rats fed the high-Ca diet compared with rats fed the low-Ca control diet on day $14(P<0 \cdot 05$; Fig. 1). Urinary excretion of Cr-EDTA by scFOS-fed rats was lower than that by control rats at the beginning of the experiment (day 2). This might represent shorter exposure of the intestinal epithelium to luminal contents due to more rapid gut transit on the scFOS diet, which has been observed in past studies ${ }^{(22)}$. From day 2, urinary Cr-EDTA excretion by scFOS-fed rats increased gradually with time compared with the control group, probably reflecting relatively slow gut microbiota adaptations due to scFOS. After 2 weeks, dietary scFOS had increased total intestinal permeability by $30 \%$ $(P<0.05$; Fig. 1).

\section{Effect of calcium and short-chain fructo-oligosaccharides on} small-intestinal permeability

Dietary effects on the lactulose:mannitol ratio were smaller and, surprisingly, in the opposite direction compared to total intestinal permeability as measured by Cr-EDTA. The lactulose:mannitol ratio on day 14 was $15 \%$ higher for rats fed the high-Ca diet $(0.23$ (SE 0.006), $P=0.0014)$ and $16 \%$ lower for rats fed the scFOS diet $(0.17$ (SE 0.009), $P=0.0038)$ compared with rats fed the low-Ca control diet (0.20 (SE 0.005)).

We also considered the lactulose and mannitol fluxes individually to gain more insight and specify whether paracellular permeability was changed. However, we did not observe significant dietary effects on individual lactulose $(P=0 \cdot 61)$ and mannitol excretion in urine $(P=0 \cdot 36$; Fig. 2). This indicates that paracellular permeability in the small intestine remained the same. In combination with the Cr-EDTA results, it can therefore be concluded that both $\mathrm{Ca}$ and scFOS affect permeability only in the colon.

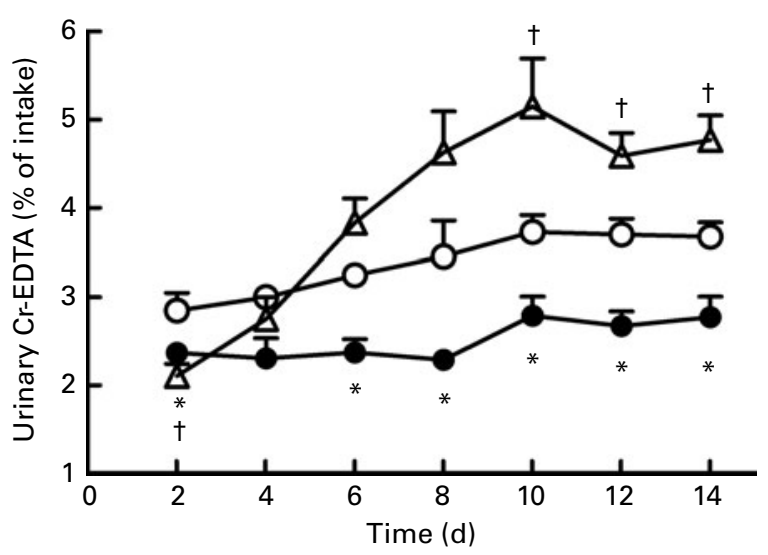

Fig. 1. Effect of dietary $\mathrm{Ca}$ and short-chain fructo-oligosaccharides (scFOS) on urinary Cr-EDTA excretion, a marker for total intestinal paracellular permeability. Results are expressed as means with their standard errors (n 13). ${ }^{*}$ Mean values of Ca-fed rats were significantly different compared with control rats $(P<0.05)$. †Mean values of scFOS-fed rats were significantly different compared with control rats $(P<0.05)$. $-\bigcirc-$, Control; $-\bullet-, \mathrm{Ca} ;-\Delta-$, scFOS. 


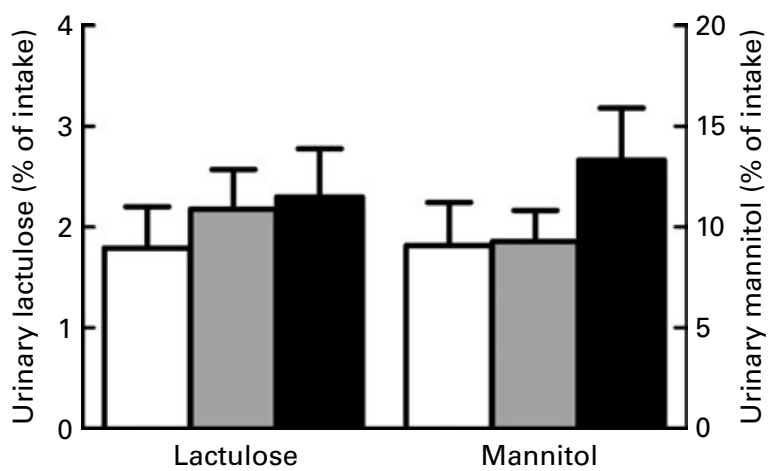

Fig. 2. Effect of dietary $\mathrm{Ca}$ and short-chain fructo-oligosaccharides (scFOS) on individual urinary lactulose and mannitol excretion. Results are expressed as means with their standard errors ( $n 13)$. $\square$, Control; $\square, \mathrm{Ca} ; \mathbf{\square}$, scFOS.

\section{Effect of faecal water from rats fed supplemental calcium or} short-chain fructo-oligosaccharides in Ussing chambers

Faecal waters were prepared from faeces of the in vivo rat experiment and tested in Ussing chambers using colonic tissue. Diet influenced the $\mathrm{pH}$ of pools of faecal water (control: 6.1; Ca: 6.5 and scFOS: 5.5). No dietary effects were observed on transepithelial resistance $(P=0.23)$, while the positive control (ethylene glycol tetraacetic acid) caused a decrease in resistance as expected $(P=0.0019$; data not shown). Also, the paracellular flux of FITC-dextran crossing the epithelial barrier was similar for all faecal waters at all time points $(P=0.67$; Fig. 3$)$. Obviously, the FITC-dextran flux was increased in the positive control due to the addition of the $\mathrm{Ca}$ chelator ethylene glycol tetraacetic acid $(P=0.0002)$.

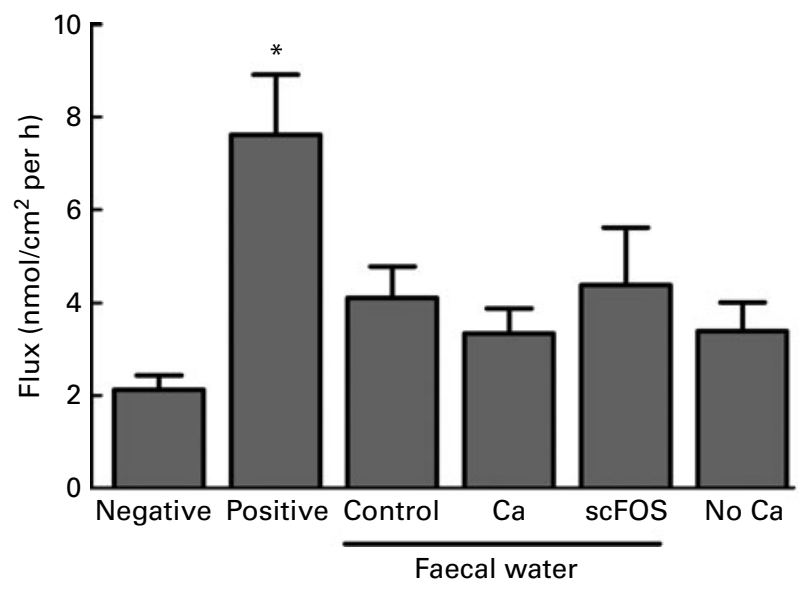

Fig. 3. Effect of faecal water from rats fed a control diet, Casupplemented diet or short-chain fructo-oligosaccharides (scFOS)supplemented diet on fluorescein isothiocyanate-labelled $4 \mathrm{kDa}$ dextran (FIFC-dextran) flux, a marker for permeability, across stripped colonic tissue in Ussing chambers. Also, the Ca-free Ringer solution at the luminal side of the Ussing chamber was studied ('no Ca'). Results (means with their standard errors) are expressed as nanomol of FITC-dextran crossing $1 \mathrm{~cm}^{2}$ of colonic epithelium in $1 \mathrm{~h}$. The interval of time shown $(60-90 \mathrm{~min})$ is representative for the whole experiment. Negative (Ringer), $n$ 12; positive (ethylene glycol tetraacetic acid in Ringer), $n$ 10; faecal water of control rats, $n$ 9; faecal water of Ca-fed rats, $n$ 10; faecal water of scFOS-fed rats, $n$ 9; no $\mathrm{Ca}(n 13) .{ }^{*}$ Mean values were significantly different compared with negative control $(P=0.0002)$.
So, diet-modified faecal waters did not influence colonic permeability in Ussing chambers. Furthermore, the Ca-free Ringer solution at the luminal side of the Ussing chamber did not influence dextran flux either ( $P=0 \cdot 09$; Fig. 3 ).

\section{Discussion}

The present study demonstrates that the effect of dietary $\mathrm{Ca}$ and scFOS on permeability in rats is localised in the colon. Supplemental Ca clearly decreased total intestinal permeability, as determined by Cr-EDTA as a marker. At the same time, it caused a less pronounced increase in the lactulose:mannitol ratio but without affecting the individual lactulose excretion, representing small-bowel permeability. Taken together, it can be concluded, although indirectly, that the overall permeability is decreased by $\mathrm{Ca}$ supplementation and that the effect is localised in the colon. The same applies for scFOS, although the results are opposite: dietary scFOS increased total intestinal permeability as measured by Cr-EDTA, whereas a minor decrease in the lactulose:mannitol ratio was observed without an effect on individual urinary lactulose values. Therefore, we can indirectly conclude that the increase in permeability caused by scFOS is also localised in the colon. The Ussing chamber experiments showed that dietary $\mathrm{Ca}$ and scFOS did not influence colonic permeability via alterations in faecal water.

The consistent finding that dietary $\mathrm{Ca}$ beneficially influences colonic permeability is very interesting. In the first place, it is intriguing that intestinal permeability apparently can be improved in a healthy situation. Secondly, an increased permeability is suggested to be a primary causative factor contributing to inflammatory bowel disease pathogenesis ${ }^{(2,23,24)}$. It is generally accepted that the gut microbiota and its intimate contact with an over-reactive immune system in the intestinal epithelium is the driving force behind the chronic inflammation $^{(25)}$. As intestinal bacteria reside mainly in the colon $^{(26)}$, decreasing of colonic permeability by a high-Ca diet might be a highly attractive nutritional intervention for inflammatory bowel disease patients. Moreover, in a previous study, we showed that supplemental $\mathrm{Ca}$ attenuated some important aspects of colitis development in HLA-B27 transgenic rats ${ }^{(11)}$.

Based on the individual lactulose results, dietary $\mathrm{Ca}$ does not influence small-intestinal permeability. The fact that Meddings \& Gibbons ${ }^{(16)}$ could not detect any lactulose and mannitol in the colon underlines the specificity of both lactulose and mannitol as markers for small-intestinal permeability. Therefore, $\mathrm{Ca}$ supplementation might only be worthwhile when targeting the colon for health improvement. The reason for the difference in localisation is unknown, but we hypothesise that $\mathrm{Ca}$ influences intestinal permeability by modulating the colonic microbiota or its metabolites. For example, Salmonella typhi and Escherichia coli, being members of the enterobacteria, are able to induce an increase in intestinal permeability ${ }^{(27)}$. In agreement with these results, dietary $\mathrm{Ca}$ decreased faecal enterobacteria in the present study (control: 9.1 (SE 0.2); Ca: $7 \cdot 3$ (SE 0.1) and scFOS: 9.1 (SE 0.1) $\log _{10}$ colony-forming units/g faeces). Effects of both $\mathrm{Ca}$ and scFOS on the intestinal microbiota have been shown before ${ }^{(8,13,14,28)}$. 
Also, the increase in total permeability caused by dietary scFOS is shown to be localised in the colon. This was hypothesised, as these carbohydrates, or prebiotics, are rapidly hydrolysed and metabolised in the colon by the endogenous microbiota $^{(29)}$. In this way, scFOS change the bacterial composition, which is often assumed to be beneficial ${ }^{(30)}$. In the present study, scFOS were supplemented, which have a degree of polymerisation ranging from 2 to 8 . Supplementation with long-chain FOS (degree of polymerisation between 2 and 60) alters the microbiota differently from supplementation with scFOS, because of differences in fermentation rate $^{(31)}$ and amount and pattern of SCFA production ${ }^{(32)}$. However, Ten Bruggencate et al. ${ }^{(8)}$ showed that scFOS and inulin (mix of short-chain and long-chain FOS; average degree of polymerisation between 10 and 12) both impaired resistance to intestinal infection in rats. On the contrary, Petersen et al. ${ }^{(33)}$ only observed increased translocation of Salmonella in mice supplemented with scFOS, and not in mice supplemented with inulin. The decreased food intake of the rats fed scFOS might be due to rapid and extensive fermentation of scFOS causing bloating and abdominal cramps ${ }^{(34)}$. These unpleasant feelings decrease appetite. As the permeability marker data are expressed as percentage of intake, the differences in food intake do not influence the outcome measurements. The Cr-EDTA excretion increased gradually with time in the scFOS group. This might reflect relatively slow gut microbiota adaptations due to scFOS. The change in the intestinal permeability by supplemental scFOS has previously been associated with a decreased resistance to intestinal infections ${ }^{(14)}$.

Paracellular permeability in the small intestine, determined by individual lactulose excretion, was not significantly influenced by the supplementation of scFOS to the diet. A similar site-specific effect of scFOS has been observed in previous studies in our laboratory. In one study, dietary scFOS increased mucosal inflammation in the caecum and colon after oral infection with Salmonella enteritidis, but not in the ileum ${ }^{(12)}$. In another study, scFOS supplementation increased mucin concentration and stimulated mucosal lactobacilli and enterobacteria in the caecum and colon, but again not in the ileum ${ }^{(14)}$. scFOS therefore clearly have region-specific effects in rats: in the colon they may exert undesirable effects, while in the small intestine no such effects were observed. Results fit with the idea that the physiological effects of scFOS are microbiota mediated and these mainly reside in the caecum and colon. Furthermore, although fermentation in the rat takes place in both the caecum and colon, the similarity of the effects of scFOS in both these organs supports the use of the rat as an appropriate model for human colonic fermentation.

Interestingly, most adverse effects of scFOS on intestinal infection, which are mentioned in this paper ${ }^{(12-14)}$, were inhibited when $\mathrm{Ca}$ was supplemented to the $\operatorname{diet}^{(8)}$. The $\mathrm{Ca}$ content of the control diet in these studies and the present investigation matches with the lower limit of the human intake range, while the Ca-supplemented diet provided more than the general habitual dietary $\mathrm{Ca}$ intake ${ }^{(11,35)}$. The dose of scFOS used in the present study $(6 \%)$ is high, but probably realistic for the human situation. Daily intake of fructosebased non-digestible carbohydrates has been estimated up to $10 \mathrm{~g}^{(36)}$. This corresponds to $2 \%$ in the diet, assuming a total food intake of $500 \mathrm{~g}$ dry weight/d. This estimation does not take into account consumption of specific meals and products supplemented with fructose-based carbohydrates, typically $3-10 \mathrm{~g} /$ portion $^{(37)}$. As the human diet still contains other non-digestible carbohydrates, supplementation with $6 \%$ is high, but certainly not unrealistic. When scFOS are fermented, SCFA are produced, which lead to acidification of the intestinal lumen. Subsequently, the protective effect of supplemental $\mathrm{Ca}$ might be due to formation of the insoluble calcium phosphate complex in the small intestine. In the colon, solubilisation of this complex increases the buffering capacity of the intestinal lumen, which may counteract the adverse effects of acidic fermentation of $\mathrm{scFOS}^{(38)}$. Indeed, the $\mathrm{pH}$ of faecal water of the rats fed the high-Ca diet was higher than that of the rats fed the low-Ca control diet. It will be interesting to investigate the effect on intestinal permeability when both $\mathrm{Ca}$ and scFOS are supplemented to the diet. This will be further explored in following studies. Finally, when studying the effects of non-digestible carbohydrates, Ca content of the diet should be taken into account.

Since the individual lactulose and mannitol excretion in urine were not influenced by the different diets, the relevance of the lactulose:mannitol ratio is questionable. Bijlsma et al. ${ }^{(39)}$ suggested that the individual lactulose and mannitol excretion values provide more information than the ratio of the two sugars. In fact, mannitol excretion in urine depends mainly on the magnitude of solvent drag caused by villus tip hyperosmolality, while urinary lactulose results truly represent paracellular permeability. By combining the two probes in a ratio, the information is lost and wrong conclusions can be drawn. For example, in the case of coeliac disease, mannitol excretion is clearly decreased because of villus atrophy, while urinary lactulose recovery is increased ${ }^{(40)}$. These findings are more informative than a ratio. In the present study, the ratio indicates that dietary effects exist, while the individual lactulose and mannitol results are not affected by the diet. Based on these findings, we conclude that small-intestinal permeability is not changed by Ca or scFOS.

In the Ussing chamber experiments, we did not observe any effect of the different faecal waters, prepared from faeces of the nutritional study, on permeability. Nevertheless, the $\mathrm{pH}$ of the faecal waters was different due to the diets. This demonstrates that fermentation of scFOS indeed influenced colonic contents, supporting the use of faecal water to study the potential scFOS effects. These Ussing chamber results suggest that the effect of $\mathrm{Ca}$ and scFOS on colonic permeability is not mediated by differences in faecal water composition. Although we observed a change in cytotoxicity of faecal water by Ca and scFOS in previous studies ${ }^{(7,12,13,41)}$, apparently luminal cytotoxicity is not responsible for the effects on colonic permeability. However, it should be kept in mind that, for example, SCFA produced by scFOS fermentation are rapidly absorbed by the colonic mucosa and therefore are largely absent in faecal waters ${ }^{(42)}$, although they might have been capable of influencing the gut wall in vivo and thus the permeability. Additionally, exposure time of the colonic tissue to the faecal water in the Ussing chamber is much shorter than that occurs in vivo. Nevertheless, we hypothesise that other mechanisms, which cannot be mimicked in the Ussing chamber, may play a role in the effects of $\mathrm{Ca}$ and scFOS on permeability. A possible 
hypothesis is that $\mathrm{Ca}$ influences the viscosity of the mucus layer, thereby altering intestinal permeability ${ }^{(43)}$. Handling the colonic tissue for use in the Ussing chambers results in partial loss of this layer, hence this effect may be missed. Moreover, as mentioned earlier and described in other papers, both $\mathrm{Ca}$ and scFOS modulate the intestinal microbiota $^{(12,28)}$, and these microbes are not present in the Ussing chambers or in the different faecal waters used in the experiments.

Although extracellular $\mathrm{Ca}$ is crucial for the maintenance of intestinal tight junction function in cell studies ${ }^{(44)}$, no direct effect of $\mathrm{Ca}$ on permeability in Ussing chambers was observed when colonic tissue was exposed to $\mathrm{Ca}$-free Ringer solution on the luminal side. These differences in observation suggest that the effect of $\mathrm{Ca}$ on permeability as observed in vivo is either indirect or long term or both.

In conclusion, the present study shows that dietary $\mathrm{Ca}$ decreases, while scFOS increase the permeability of the rat colon. We hypothesise that modulation of mucins and/or microbiota is important for the permeability effects of both $\mathrm{Ca}$ and scFOS, since in vivo results could not be mimicked in Ussing chambers. The results of the present study emphasise that nutrition can play an important role in the modulation of gut epithelial integrity.

\section{Acknowledgements}

The authors wish to thank the biotechnicians at the Small Animal Centre (Wageningen University and Research Centre, The Netherlands) for their expert assistance. The present study was supported by TI Food and Nutrition (Wageningen, The Netherlands). None of the authors has conflicts of interest. M. A. A. S. contributed to study design, experimental procedures, data analysis, data interpretation and manuscript writing. A. R. contributed to study design, experimental procedures and data interpretation. A. J. S. and C. V. assisted in experimental procedures and data analysis. R.-J. M. B. contributed to data interpretation and assisted in manuscript writing. L. E. M. W. contributed to data interpretation and manuscript writing. R. v. d. M. and I. M. J. B.-O. contributed to study design, data interpretation and manuscript writing.

\section{References}

1. Farhadi A, Banan A, Fields J, et al. (2003) Intestinal barrier: an interface between health and disease. $J$ Gastroenterol Hepatol 18, 479-497.

2. Arrieta MC, Bistritz L \& Meddings JB (2006) Alterations in intestinal permeability. Gut 55, 1512-1520.

3. Groschwitz KR \& Hogan SP (2009) Intestinal barrier function: molecular regulation and disease pathogenesis. J Allergy Clin Immunol 124, 3-20; quiz 21-22.

4. Spiller RC (2007) Role of infection in irritable bowel syndrome. J Gastroenterol 42, Suppl. 17, 41-47.

5. Clayburgh DR, Shen L \& Turner JR (2004) A porous defense: the leaky epithelial barrier in intestinal disease. Lab Invest $\mathbf{8 4}$, 282-291.

6. Bovee-Oudenhoven IM, Lettink-Wissink ML, Van Doesburg W, et al. (2003) Diarrhea caused by enterotoxigenic Escherichia coli infection of humans is inhibited by dietary calcium. Gastroenterology 125, 469-476.
7. Bovee-Oudenhoven IM, Termont DS, Weerkamp AH, et al. (1997) Dietary calcium inhibits the intestinal colonization and translocation of Salmonella in rats. Gastroenterology 113, $550-557$.

8. Ten Bruggencate SJ, Bovee-Oudenhoven IM, Lettink-Wissink ML, et al. (2004) Dietary fructo-oligosaccharides and inulin decrease resistance of rats to Salmonella: protective role of calcium. Gut 53, 530-535.

9. Govers MJ, Termont DS, Lapre JA, et al. (1996) Calcium in milk products precipitates intestinal fatty acids and secondary bile acids and thus inhibits colonic cytotoxicity in humans. Cancer Res 56, 3270-3275.

10. Lapre JA, De Vries HT, Koeman JH, et al. (1993) The antiproliferative effect of dietary calcium on colonic epithelium is mediated by luminal surfactants and dependent on the type of dietary fat. Cancer Res 53, 784-789.

11. Schepens MA, Schonewille AJ, Vink C, et al. (2009) Supplemental calcium attenuates the colitis-related increase in diarrhea, intestinal permeability, and extracellular matrix breakdown in HLA-B27 transgenic rats. J Nutr 139, $1525-1533$.

12. Bovee-Oudenhoven IM, Ten Bruggencate SJ, Lettink-Wissink ML, et al. (2003) Dietary fructo-oligosaccharides and lactulose inhibit intestinal colonisation but stimulate translocation of Salmonella in rats. Gut 52, 1572-1578.

13. Ten Bruggencate SJ, Bovee-Oudenhoven IM, Lettink-Wissink ML, et al. (2003) Dietary fructo-oligosaccharides dosedependently increase translocation of salmonella in rats. $J$ Nutr 133, 2313-2318.

14. Ten Bruggencate SJ, Bovee-Oudenhoven IM, Lettink-Wissink ML, et al. (2005) Dietary fructooligosaccharides increase intestinal permeability in rats. $J$ Nutr $\mathbf{1 3 5}, 837-842$.

15. Aabakken L (1989) Cr-ethylenediaminetetraacetic acid absorption test. Methodologic aspects. Scand J Gastroenterol 24, 351-358.

16. Meddings JB \& Gibbons I (1998) Discrimination of sitespecific alterations in gastrointestinal permeability in the rat. Gastroenterology 114, 83-92.

17. Reeves PG, Nielsen FH \& Fahey GC Jr (1993) AIN-93 purified diets for laboratory rodents: final report of the American Institute of Nutrition ad hoc writing committee on the reformulation of the AIN-76A rodent diet. J Nutr 123, 1939-1951.

18. Arslan G, Atasever T, Cindoruk M, et al. (2001) (51)CrEDTA colonic permeability and therapy response in patients with ulcerative colitis. Nucl Med Commun 22, 997-1001.

19. Binnerts W, Van het Klooster A \& Frens A (1968) Soluble chromium indicator measured by atomic absorption in digestion experiments. Vet Rec 82, 470.

20. Bjarnason I (1994) Intestinal permeability. Gut 35, S18-S22.

21. Sesink AL, Termont DS, Kleibeuker JH, et al. (1999) Red meat and colon cancer: the cytotoxic and hyperproliferative effects of dietary heme. Cancer Res 59, 5704-5709.

22. Hansen I, Knudsen KE \& Eggum BO (1992) Gastrointestinal implications in the rat of wheat bran, oat bran and pea fibre. Br J Nutr 68, 451-462.

23. Arnott ID, Kingstone K \& Ghosh S (2000) Abnormal intestinal permeability predicts relapse in inactive Crohn disease. Scand J Gastroenterol 35, 1163-1169.

24. Wyatt J, Vogelsang H, Hubl W, et al. (1993) Intestinal permeability and the prediction of relapse in Crohn's disease. Lancet 341, 1437-1439.

25. Sartor RB (2006) Mechanisms of disease: pathogenesis of Crohn's disease and ulcerative colitis. Nat Clin Pract Gastroenterol Hepatol 3, 390-407.

26. Guarner F \& Malagelada JR (2003) Gut flora in health and disease. Lancet 361, 512-519. 
27. Mangell P, Nejdfors P, Wang M, et al. (2002) Lactobacillus plantarum $299 \mathrm{v}$ inhibits Escherichia coli-induced intestinal permeability. Dig Dis Sci 47, 511-516.

28. Bovee-Oudenhoven IM, Wissink ML, Wouters JT, et al. (1999) Dietary calcium phosphate stimulates intestinal lactobacilli and decreases the severity of a Salmonella infection in rats. J Nutr 129, 607-612.

29. Andersson HB, Ellegard LH \& Bosaeus IG (1999) Nondigestibility characteristics of inulin and oligofructose in humans. J Nutr 129, 1428S-1430S

30. Gibson GR \& Roberfroid MB (1995) Dietary modulation of the human colonic microbiota: introducing the concept of prebiotics. J Nutr 125, 1401-1412.

31. Roberfroid MB, Van Loo JA \& Gibson GR (1998) The bifidogenic nature of chicory inulin and its hydrolysis products. J Nutr $\mathbf{1 2 8}$, $11-19$.

32. Macfarlane S \& Macfarlane GT (2003) Regulation of shortchain fatty acid production. Proc Nutr Soc 62, 67-72.

33. Petersen A, Heegaard PM, Pedersen AL, et al. (2009) Some putative prebiotics increase the severity of Salmonella enterica serovar Typhimurium infection in mice. $B M C$ Microbiol 9, 245

34. Briet F, Achour L, Flourie B, et al. (1995) Symptomatic response to varying levels of fructo-oligosaccharides consumed occasionally or regularly. Eur J Clin Nutr 49, 501-507.

35. Alaimo K, McDowell MA, Briefel RR, et al. (1994) Dietary intake of vitamins, minerals, and fiber of persons ages 2 months and over in the United States: Third National Health and Nutrition Examination Survey, Phase 1, 1988-91. Adv Data, 1-28.
36. Coussement PA (1999) Inulin and oligofructose: safe intakes and legal status. J Nutr 129, 1412S-1417S

37. van Loo J, Coussement P, de Leenheer L, et al. (1995) On the presence of inulin and oligofructose as natural ingredients in the western diet. Crit Rev Food Sci Nutr 35, $525-552$.

38. Remesy C, Levrat MA, Gamet L, et al. (1993) Cecal fermentations in rats fed oligosaccharides (inulin) are modulated by dietary calcium level. Am J Physiol 264, G855-G862.

39. Bijlsma PB, Peeters RA, Groot JA, et al. (1995) Differential in vivo and in vitro intestinal permeability to lactulose and mannitol in animals and humans: a hypothesis. Gastroenterology 108, 687-696.

40. Ukabam SO \& Cooper BT (1984) Small intestinal permeability to mannitol, lactulose, and polyethylene glycol 400 in celiac disease. Dig Dis Sci 29, 809-816.

41. Govers MJ \& Van der Meer R (1993) Effects of dietary calcium and phosphate on the intestinal interactions between calcium, phosphate, fatty acids, and bile acids. Gut $\mathbf{3 4}$, 365-370.

42. Alles MS, Hautvast JG, Nagengast FM, et al. (1996) Fate of fructo-oligosaccharides in the human intestine. Br J Nutr 76, 211-221.

43. Crowther RS, Marriott C \& James SL (1984) Cation induced changes in the rheological properties of purified mucus glycoprotein gels. Biorheology 21, 253-263.

44. Gonzalez-Mariscal L, Contreras RG, Bolivar JJ, et al. (1990) Role of calcium in tight junction formation between epithelial cells. Am J Physiol 259, C978-C986. 